\title{
Dual-camera high-speed imaging of the ignition modes of ethanol, methanol and n-hexane in a shock tube
}

\author{
M. Figueroa-Labastida ${ }^{1}$, Jihad Badra ${ }^{2}$, A. Farooq ${ }^{1 *}$ \\ ${ }^{1}$ King Abdullah University of Science and Technology, Clean Combustion Research Center, Physical \\ Sciences and Engineering Division, Thuwal 23955-6900, Saudi Arabia \\ ${ }^{2}$ Fuel Technology Division, R\&DC, Saudi Aramco, Dhahran, Saudi Arabia
}

Corresponding author email: aamir.farooq@kaust.edu.sa

\begin{abstract}
Shock tubes are used as homogeneous batch reactors to measure ignition delay times, reaction rate coefficients and species time-histories of a variety of chemical systems. Any non-ideality or inhomogeneity in the shock tube experiment would affect the quality and usefulness of measured data. Experimental and computational efforts have previously been carried out to characterize the regimes of ideal operation of shock tubes. High-speed imaging has proven to be a highly useful tool to assess ignition homogeneity. In this work, a dual-camera setup has been used with an optical end-section in a circular shock tube to obtain simultaneous high-speed images from the shock tube endwall and sidewall, thus providing visualization of the ignition phenomenon in three dimensions. Two case studies are presented herein to demonstrate the quality of data and insights that are obtained using this diagnostic technique to study the ignition modes of different fuels. The first is a comparison of the ignition of two alternative fuels, methanol and ethanol, and the second is a study of the ignition dependence on the fuel concentration of a representative paraffinic fuel, $n$-hexane. The unique dual-camera imaging diagnostic enabled deeper insights into the ignition homogeneity, with all fuels exhibiting localized ignition at low temperatures. Methanol showed a higher propensity than ethanol to ignite far from the endwall, and the high concentration of $n$-hexane led to inhomogeneous ignition.
\end{abstract}

Keywords: Shock tube; high-speed imaging; ignition modes; ethanol; methanol; n-hexane. 


\section{Introduction}

Shock tubes are very important tools in several areas of combustion research. The measurement of rate coefficients and ignition delay times in shock tubes provide highly valuable data for the development and validation of chemical kinetic models. Chemical phenomena are usually observed near the shock tube endwall, either behind the incident or the reflected shock wave. Shock tubes are commonly assumed to undergo homogeneous reaction. Nevertheless, some recent works have reported that non-idealities may affect ignition measurements. Javed et al. [1] reported unexpectedly expedited ignition delay times (IDTs), in comparison to well-validated chemical kinetic models, of $n$-heptane and $n$-hexane in the intermediate temperature regime. Uygun et al. [2] observed preignition pressure rise during the combustion of 2methylfuran and confirmed the existence of deflagrative flame kernels, via schlieren imaging, preceding the main ignition event. Recent works of Pinzón et al. [3] and Mathieu et al. [4] observed early ignition events, resulting in expedited IDTs at low temperatures for methanol and ethanol, respectively. Numerical simulations have also been employed to investigate possible causes of such unexpected behavior. Grogan

et al. [5] concluded that temperature non-homogeneities may be caused by the interaction of reflected shock wave with the boundary layer, and Santner et al. [6] identified heat transfer characteristics and kinetic sensitivity of the mixture as the key parameters affecting the perseverance of hot spots.

In the light of observed non-idealities in ignition measurements using shock tubes, high-speed imaging has been applied to identify non-ideal events and determine possible mechanisms of such sightings. Our previous work on ethanol mixtures [7] utilized high-speed imaging from a transparent endwall to understand ignition modes of ethanol at different conditions, where inhomogeneous ignition was observed at lower temperatures and higher ethanol concentration. Other shock tube endwall imaging studies are found in literature. Troutman et al. [8] recorded $\mathrm{OH}^{*}$ chemiluminescence images for $n$-heptane combustion and identified the cleanliness and internal surface disruptions as important parameters 
promoting non-homogeneous ignition. Tulgestke et al. [9] described the existence and accumulation of diaphragm particles in combustion experiments. Ninnemann et al. [10] studied non-homogenous ignition for $\mathrm{H}_{2} / \mathrm{O}_{2}$ system and found good correlation between their observations and the thermal diffusivity of the mixtures. Pryor et al. [11] studied the ignition of methane under high $\mathrm{CO}_{2}$ dilution.

Shock tube endwall imaging, which has recently been implemented in a few laboratories, permits the visual characterization of ignition in the radial direction. Nevertheless, it can neither determine axial location of the observed features with respect to the endwall nor the speed of axial propagation of the reaction fronts. Ignition occurring far from the endwall was reported in previous studies which utilized imaging in rectangular section shock tubes with lateral windows [12-15]. However, these studies are limited, as the transition of shock waves from circular to square section can generate undesired pressure/rarefaction waves and cold/hot spots. Lee et al. [13] and Wang et al. [16] implemented a slit window circular end-section in their shock tube for schlieren imaging of ethanol and $\mathrm{H}_{2} /$ air/steam combustion, respectively. More recently, Ninnemann et al. [17] introduced the initial description of a fully optically accessible test section in a circular shock tube, utilizing a quartz annulus fused to a transparent endwall, and showed preliminary two-camera measurements during iso-octane oxidation. Nativel et al. [18] examined the use of a lateral endoscopic imaging setup as a complement to traditional endwall imaging for the investigation of ethanol oxidation at 20 bar. Figueroa-Labastida and Farooq [19] reported the implementation of a simultaneous lateral and endwall high-speed visualization diagnostic in a circular shock tube. They showed demonstrative imaging measurements via $142 \mathrm{~mm}$ diameter endwall and 208 $\mathrm{mm}$ long sidewall windows with resolutions of $0.17 \mathrm{~mm} /$ pixel and $0.26 \mathrm{~mm} /$ pixel, respectively.

In this work, the simultaneous lateral and endwall imaging diagnostic [19] has been applied to carry out detailed investigation of the ignition modes of various fuels. We have studied two distinct cases; the first being a comparison between the ignition of two alcohols, methanol and ethanol, and the second focused 
on the effects of fuel concentration on the ignition of $n$-hexane. Our objective is to extract, analyze and compare ignition features that can be measured from the dual-camera visualization diagnostic, such as the location of ignition features and their propagation speeds.

\section{Experimental Setup}

Experiments were performed in the Chemical Kinetics Shock Tube facility at King Abdullah University of Science and Technology (KAUST). The driver and driven sections of this shock tube are each $9.1 \mathrm{~m}$ long, separated by a polycarbonate diaphragm, with an inner diameter of $14.2 \mathrm{~cm}$. Facility and instrumentation details are described previously [7]. Methanol (99.8\%), ethanol (> 99.8\%) and $n$-hexane (99\%) were obtained from Sigma-Aldrich; oxygen and argon (99.999\%) were supplied by Air Liquide.

A new circular optical end-section has recently been designed and tested [19]; maximum operation pressure of the optical section is 30 bar. This section allows simultaneous axial (from sidewall slit window) and radial (from endwall window) imaging during the ignition process. Two monochrome Photron Fastcam SA-X2 high-speed cameras, each equipped with a UV lens of $105 \mathrm{~mm}$ focal length and optimized transmissivity between $250-650 \mathrm{~nm}$, were synchronized to record the natural luminosity of combustion at 50,000 frames per second. Spatial resolution is $0.26 \mathrm{~mm} / \mathrm{pixel}$ and $0.17 \mathrm{~mm} /$ pixel for sidewall and endwall images, respectively. Although the slit window is $300 \mathrm{~mm}$ long, axial length shown in sidewall images is limited to $208 \mathrm{~mm}$ due to spatial constraints. The experimental setup is shown in Fig. 1, and is same as reported previously [19]. 


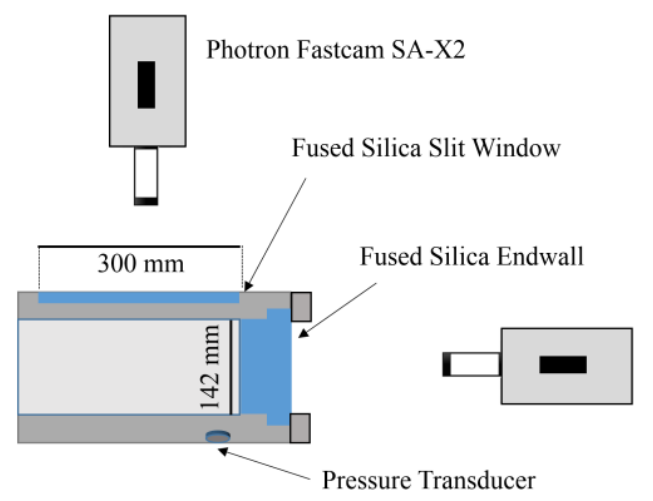

Figure 1. Optical end section for simultaneous lateral and endwall visualization.

\section{Results and Discussion}

The dual-camera imaging experiments were performed to study ignition modes of various fuels over a range of experimental conditions. Two alcohol fuels, methanol and ethanol, have recently been studied in

the context of non-homogenous ignition $[3,4,7,13,20-24]$. Here, we have investigated the ignition of these two alcohols with the help of our unique imaging diagnostic. Secondly, in contrast to these alcoholic fuels exhibiting little low-temperature reactivity, we have examined the ignition of a fuel with NTC behavior, i.e., $n$-hexane, wherein $n$-hexane mixtures with different levels of dilution are contrasted.

\subsection{Non-homogeneity in Methanol and Ethanol Ignition}

Ethanol has been identified as a fuel with high tendency to undergo preignition due to its high burning velocity, thus low laminar flame thickness $[13,20]$ and this has been observed in shock tube $[7,13,21]$ and rapid compression machine experiments [22, 23] in the form of expedited IDTs and the visualization of non-homogeneous ignition. A correlation between the appearance of ignition non-idealities and thermal diffusivity of mixtures was first introduced by Walton et al. [25] in their study of iso-octane ignition. In our recent work on ethanol [7], we could not find a conclusive evidence that thermal diffusivity is the primary factor to describe the preignition tendency of ethanol. A good correlation was found with the 
laminar flame thickness $(\delta)$ of various mixtures, based on the criterion proposed by Kalghatgi et al. [20], wherein a mixture with smaller $\delta$ would be more prone to sustain ignition in the presence of a hot spot.

Recently, methanol has been reported to show unexpected behavior during IDT measurements in a shock tube [3] and a rapid compression machine [24], with sporadic cases of significantly expedited ignition. Methanol is thus an interesting fuel for direct comparison to ethanol for the previously observed phenomena [7]. Here, we compare ignition modes of ethanol and methanol using the two-camera setup which not only enables us to assess ignition homogeneity but also permits the observation of the ignition location and the speed of propagating fronts. We chose various mixtures of methanol and ethanol which may be expected to exhibit non-homogeneous ignition based on the laminar flame thickness criterion [20] and are summarized in Table 1.

Table 1. Composition of studied gas mixtures.

\begin{tabular}{|c|c|c|c|c|c|c|}
\hline Fuel & Mixture & $\begin{array}{c}\mathrm{X}_{\text {Fuel }} \\
{[\%]}\end{array}$ & $\begin{array}{c}\mathrm{X}_{\mathrm{O} 2} \\
{[\%]}\end{array}$ & $\begin{array}{c}\mathrm{X}_{\mathrm{Ar}} \\
{[\%]}\end{array}$ & $\begin{array}{c}\mathrm{X}_{\mathrm{N} 2} \\
{[\%]}\end{array}$ & $\phi$ \\
\hline Methanol & $\mathrm{A}$ & 12.3 & 18.4 & 69.3 & 0.0 & 1.0 \\
\cline { 2 - 7 } & $\mathrm{B}$ & 12.3 & 18.4 & 0.0 & 69.3 & 1.0 \\
\cline { 2 - 7 } & $\mathrm{C}$ & 5.0 & 15.0 & 80.0 & 0.0 & 0.5 \\
\hline Ethanol & $\mathrm{D}$ & 5.0 & 15.0 & 80.0 & 0.0 & 1 \\
\cline { 2 - 7 } & $\mathrm{E}$ & 5.0 & 30.0 & 65.0 & 0.0 & 0.5 \\
\hline
\end{tabular}

Figure 2 shows calculated laminar flame thickness $(\delta)$ as a function of temperature for selected mixtures of ethanol and methanol. These calculations were carried out using Chemkin-Pro with AramcoMech 3.0 model [26]. Kalghatgi et al. [20] described the flame thickness of a mixture to be indicative of the probability of the growth of a flame kernel. The lower this value is, the higher the chance that a hot spot would have a larger size than the critical flame thickness, which would allow the kernel to be sustained and posteriorly develop into a deflagration wave. Therefore, a mixture with low value of flame thickness 
would be more prone to preignition. Considering this, mixture A and mixture E, shown in Fig. 2 as having the lowest values of $\delta$, are the candidates to present non-homogeneous ignition. Additional mixtures with higher values of flame thickness, such as mixtures B, C and D were selected in order to contrast the observed ignition modes.

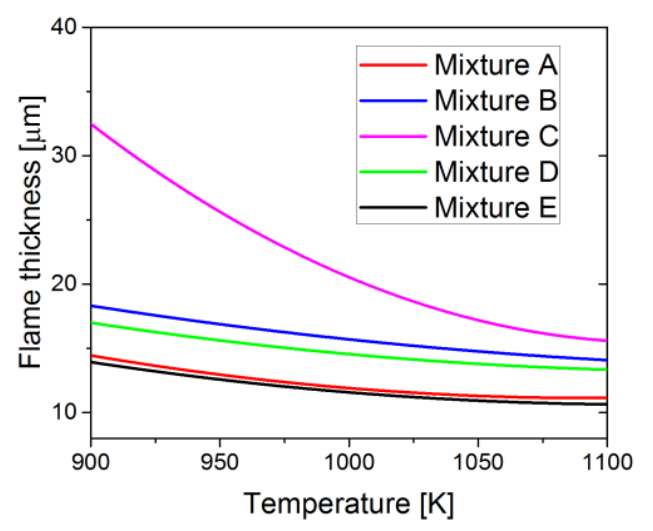

Figure 2. Calculated laminar flame thickness of different mixtures of ethanol and methanol.

\subsection{Imaging of Methanol and Ethanol Ignition}

High-speed images have been recorded for the proposed methanol and ethanol mixtures at different conditions of temperature and pressure, and will be discussed in this section. Figure 3 shows a comparison between the non-idealities observed in methanol and ethanol ignition. Each panel shows the circular image, visualized from the endwall, and the respective lateral image visualized from the sidewall slit window. In lateral images, the leftmost edge is the endwall $(\mathrm{x}=0)$ and the rightmost edge is at $208 \mathrm{~mm}$ from the endwall. All leftover diaphragm particles in the optical section were removed after each run, and the optical section was cleaned thoroughly with acetone. The rest of the driven section was not cleaned to mimic the usual continuous operation of a shock tube. The cases shown in Fig. 3 are chosen to have nominally similar ignition delay duration. 


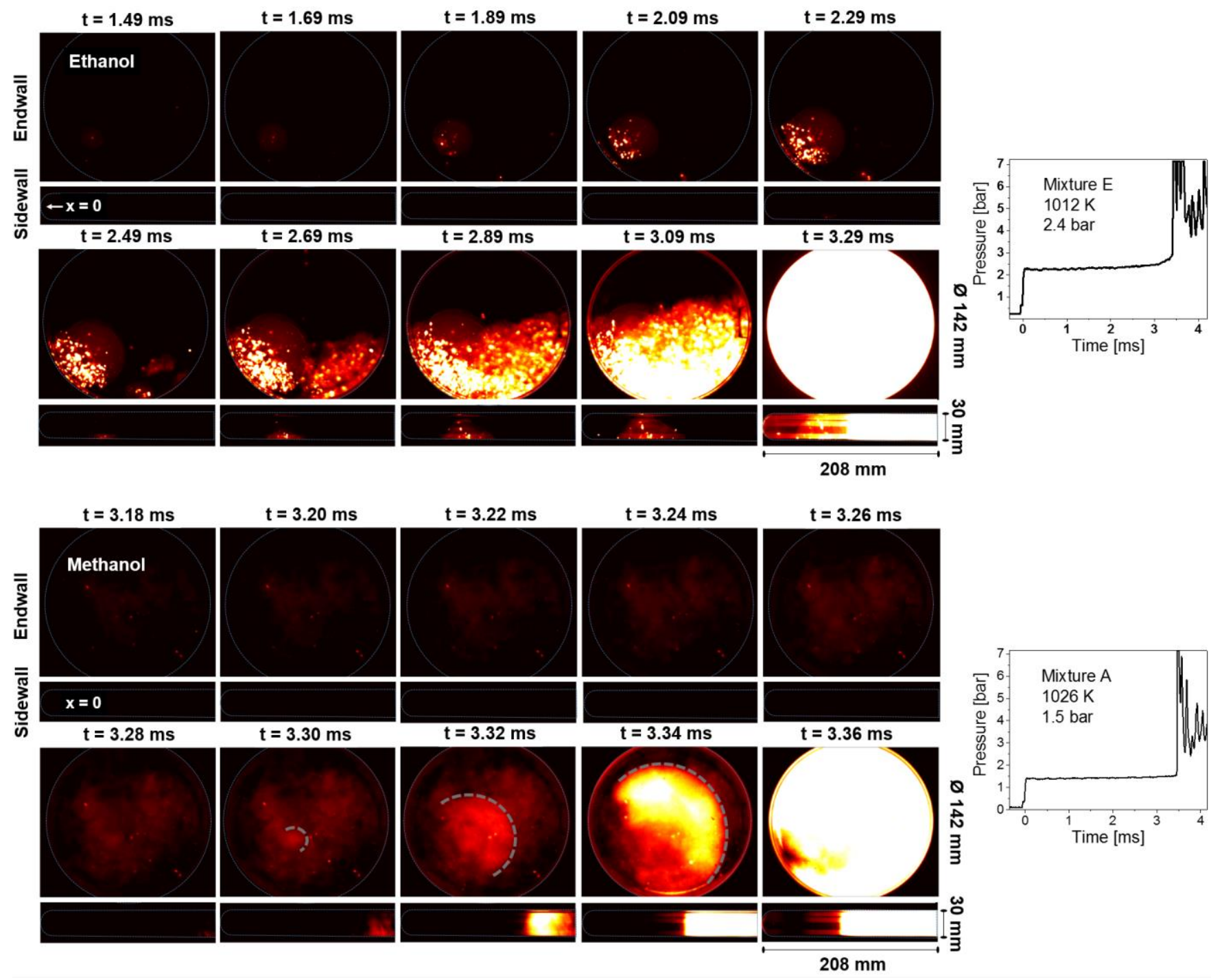

Figure 3. Simultaneous endwall and sidewall imaging of ethanol mixture $\mathrm{E}$ at $1012 \mathrm{~K}$ and $2.4 \mathrm{bar}$ and methanol mixture $\mathrm{A}$ at $1026 \mathrm{~K}$ and $1.5 \mathrm{bar}$. Indicated times for each frame refer to the time after the passage of reflected shock wave $(t=0)$. Endwall is located at $\mathrm{x}=0$. Pressure traces measured with a Kistler pressure transducer at a sidewall port ( $2 \mathrm{~cm}$ from endwall) are also shown.

Ethanol, as described in our previous work [7], exhibited non-homogeneous ignition for fuel-lean $(\phi=$ 0.5) mixture E at relatively low temperatures. In Fig. 3, endwall imaging of ethanol oxidation shows the appearance of an emission feature near the lower left part of the circular section. This can be observed from times as early as $1.49 \mathrm{~ms}$ and slowly grows until there is a second feature observed from the bottom right which thrives in a more rapid way and seems to combine with the first feature to develop into the 
main ignition. If only endwall imaging is used, one may assume that these features are developing near the endwall and it will not be possible to determine the axial location of these emission features. The implementation of additional imaging through the sidewall lateral window allows us to visualize that the first feature is appearing at $\sim 73 \mathrm{~mm}$ from the endwall, while the second feature is being developed even farther than the observation length of $208 \mathrm{~mm}$. This second feature travels towards the endwall (see the sidewall image at $t=3.29 \mathrm{~ms}$ ) while the circular image is already filled up with light. Therefore, in this case, ignition is happening quite far from the endwall. Both the existence of an emission kernel growing close to the endwall and the main ignition happening far from the endwall contribute to the preignition energy release that characterized the behavior of these ethanol mixtures in our previous work [7]. Many bright spots can be observed inside the deflagrative emission spots. These may be related to the presence of diaphragm particles in the driven section. Tulgestke et al. [9] described that small polycarbonate particle would exist even after thorough cleaning of the shock tube. Experiments in additional shock tube facilities $[10,27]$ have also observed these and pointed out that the presence of these particles did not affect the measurements of ignition delay times.

The methanol mixture shown in Fig. 3 has a relatively low value of flame thickness and, therefore, may be expected to show non-ideal ignition behavior. At early times, endwall imaging shows distributed emission in the central section which starts growing in both size and emission intensity. However, before this feature could develop into ignition, it is dominated by a quick and strong process that inhibits its influence on the final ignition. This second front, marked with dashed semi-circled lines, can be identified as a sphere-like propagation between the 3.32 and $3.34 \mathrm{~ms}$ frames. Sidewall imaging shows that the strong ignition starts somewhere around $194 \mathrm{~mm}$ from the endwall. Because of these characteristics, the observed process is classified to be intermediate between a localized and a rapid / homogeneous process. The pressure traces for these experiments are also presented in Fig. 3, where a small preignition pressure rise 
can be identified for the ethanol case but a nearly ideal pressure trace is seen for the methanol case. This shows that pressure measurements, performed close to the endwall, alone may be insufficient to identify far-wall ignition / non-homogeneities in shock tube experiments of relatively long test times. Far-wall ignition is defined here to be an ignition initiating farther than $2.0 \mathrm{~cm}$ (location of Kistler pressure transducer) from the endwall of the shock tube. Figure 4 shows the combustion of a methanol mixture at a lower temperature of $941 \mathrm{~K}$ and 1.7 bar. At these conditions, the mixture exhibits an ignition process which is started by localized emission spots which propagate as deflagrations until main ignition is achieved. Two of these localized features are delimited with dashed lines in Fig. 4. Additionally, sidewall imaging shows this process is a far-wall ignition occurring around $208 \mathrm{~mm}$ away from the endwall, therefore this process is classified as localized / non-homogeneous ignition. A preignition pressure rise can be identified in the pressure trace for this case, which is noticeable $\sim 6 \mathrm{~ms}$ after the passage of the reflected shock wave. 

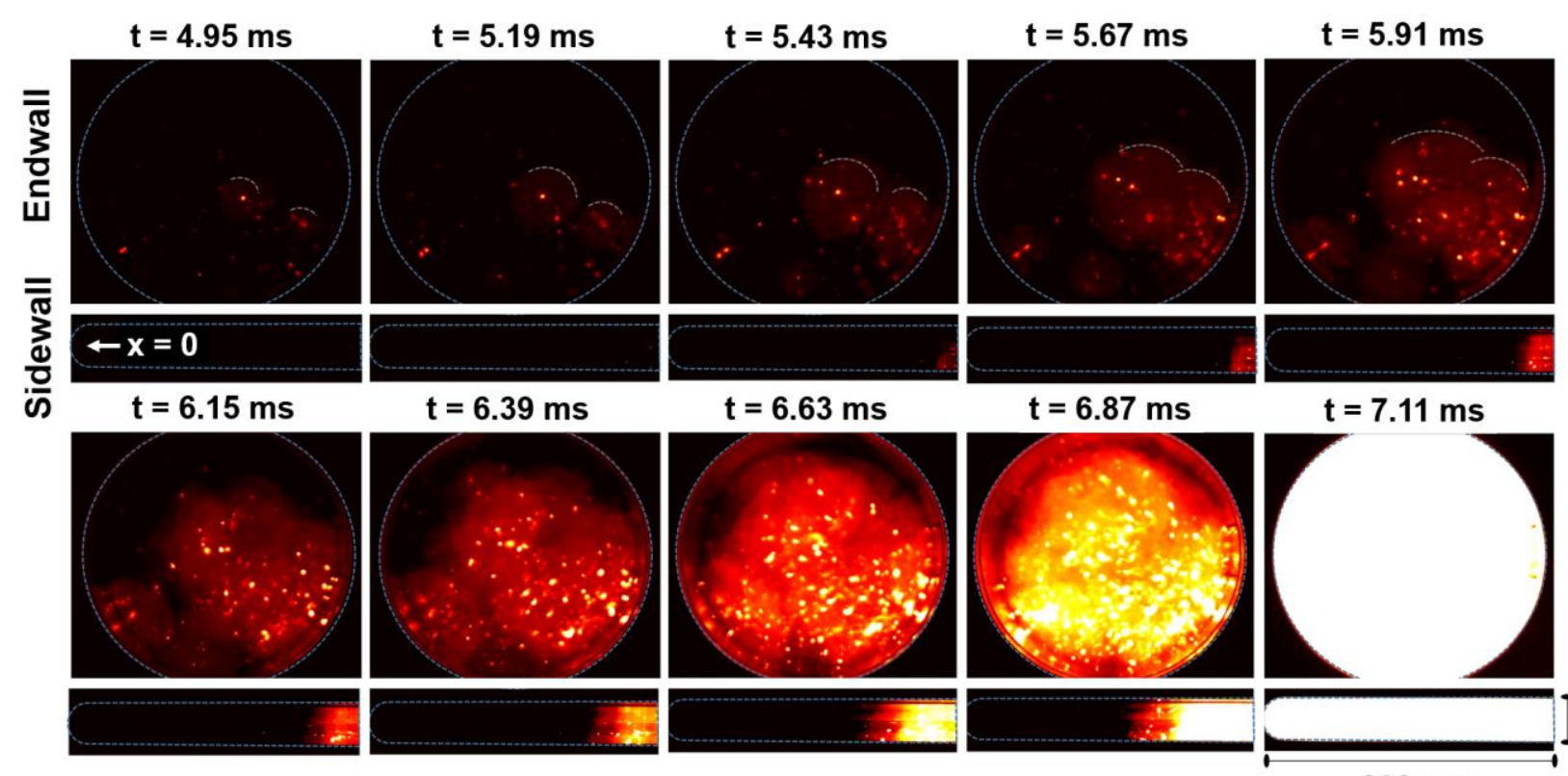

$\mathrm{t}=7.11 \mathrm{~ms}$

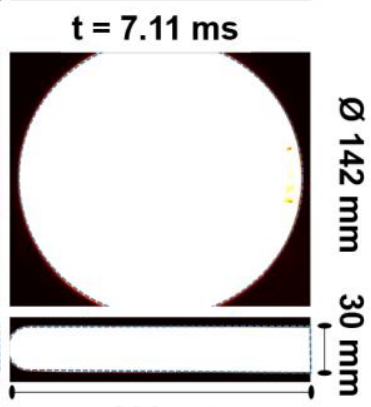

$208 \mathrm{~mm}$

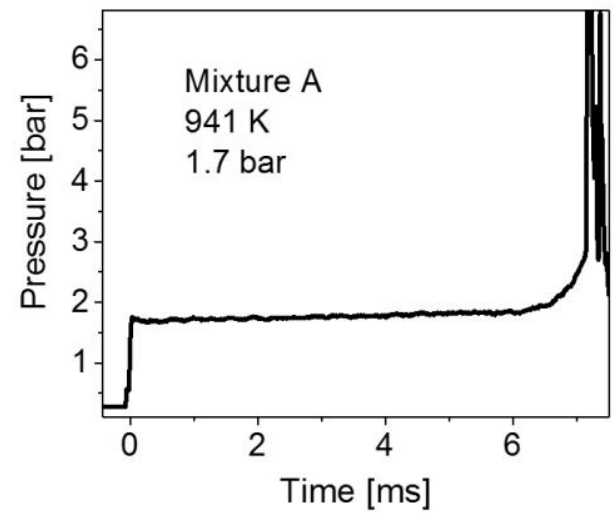

Figure 4. Simultaneous endwall and sidewall imaging of methanol mixture A at $941 \mathrm{~K}$ and 1.7 bar. Indicated times refer to the time after the passage of reflected shock wave $(t=0)$. Endwall is located at $x=0$. Pressure trace measured with a Kistler pressure transducer at a sidewall port $(2 \mathrm{~cm}$ from endwall) is also shown.

Table 2 summarizes the mixtures and experimental conditions tested for ethanol and methanol. The location of observed ignition features, which would be expected to be $\mathrm{x}=0$ for ideal experiments, and the velocity of reaction fronts are presented. Both mixture A and E show the presence of low-velocity initial features that develop until an additional fast front appears at a time closer to the overall measured ignition delay time (e.g., in Fig. 3 the fast front appears $900 \mu$ s before the total ignition of ethanol and $150 \mu \mathrm{s}$ before the total ignition of methanol) or until the localized emission spots promote the overall ignition 
(e.g., see methanol ignition at $941 \mathrm{~K}$ in Fig. 4). To characterize these events, two different front propagation speeds, calculated from the endwall and sidewall imaging, are listed in Table 2 . These speeds were graphically estimated from the images, using a fixed origin point at time zero and the respective displacement to a point $(r)$ in time $t$. Initial speed of propagation of the emission fronts is calculated using approximately 5 frames after the first identification of emission, while speeds close to ignition are extracted from 2 or 3 frames due to the process being very rapid near ignition. The estimated uncertainty of the reported speeds is approximately $\pm 10 \%$, which arises from temporal and spatial resolution constrains in the measured images. For cases where the illuminated feature evolved in a fast manner which did not permit the identification of a clear displacement position $(r)$, it was branded as fast ignition. By comparing the velocities of the observed fronts to the speed of sound ( $\mathrm{v}_{\text {sound }}$ ) of the unburned mixture, it is evident that early emission kernels propagate in a subsonic fashion. The posterior rapid front, which ignites the reactive mixtures, propagates supersonically. Ethanol mixture E has measured front speeds close (within $\pm 10 \%$ ) to their theoretical Chapman-Jouguet velocity $\left(\mathrm{v}_{\mathrm{CJ}}\right)$ at low temperatures; thus, a detonation wave is likely to be formed. On the other hand, methanol mixture A has lower front speeds compared to their $\mathrm{v}_{\mathrm{CJ}}$. 
Table 2. Summary of dual-camera imaging experiments: methanol and ethanol

\begin{tabular}{|c|c|c|c|c|c|c|c|c|c|c|}
\hline \multirow[t]{2}{*}{$\mathrm{T}[\mathrm{K}]$} & \multirow{2}{*}{$\begin{array}{c}\mathrm{P} \\
\text { [bar] }\end{array}$} & \multicolumn{2}{|c|}{$\dot{x}[\mathrm{~m} / \mathrm{s}]$} & \multicolumn{2}{|c|}{$\mathrm{v}[\mathrm{m} / \mathrm{s}]$} & \multirow[t]{2}{*}{$\mathrm{x}_{0}[\mathrm{~mm}]$} & \multirow{2}{*}{$\begin{array}{l}\mathrm{V}_{\mathrm{sound}} \\
{[\mathrm{m} / \mathrm{s}]}\end{array}$} & \multirow{2}{*}{$\begin{array}{c}\mathrm{v}_{\mathrm{CJ}} \\
{[\mathrm{m} / \mathrm{s}]}\end{array}$} & \multirow{2}{*}{$\begin{array}{c}\boldsymbol{\tau}_{\mathrm{nh}} \\
{[\mu \mathrm{s}]}\end{array}$} & \multirow[t]{2}{*}{ Homogeneity } \\
\hline & & $\begin{array}{l}\text { First } \\
\text { feature }\end{array}$ & $\begin{array}{c}\text { At } \\
\text { ignition }\end{array}$ & $\begin{array}{c}\text { First } \\
\text { feature }\end{array}$ & $\begin{array}{c}\text { At } \\
\text { ignition }\end{array}$ & & & & & \\
\hline \multicolumn{11}{|c|}{ Mixture A methanol / $\mathrm{O}_{2} / \mathrm{Ar}\left(\varphi=1 ; \mathrm{O}_{2}: \mathrm{Ar}=1: 3.76\right)$} \\
\hline 941 & 1.7 & 27 & 1399 & 35 & 1380 & $\sim 208$ & 532 & 1677 & 2515 & Localized \\
\hline 1026 & 1.5 & 544 & 1468 & 286 & 1371 & 198 & 554 & 1666 & 277 & Intermediate \\
\hline 1112 & 1.34 & 438 & 1446 & 536 & 1462 & 49 & 577 & 1655 & 109 & Homogeneous \\
\hline \multicolumn{11}{|c|}{ Mixture B methanol / $\mathbf{O}_{2} / \mathbf{N}_{\mathbf{2}}\left(\varphi=1 ; \mathrm{O}_{2}: \mathrm{N}_{2}=1: 3.76\right)$} \\
\hline 1026 & 2 & 138 & 1743 & 118 & 1838 & $>208$ & 606 & 1783 & 331 & Intermediate \\
\hline 1061 & 1.9 & 175 & 1381 & 304 & 908 & 123 & 616.5 & 1780 & 132 & Homogeneous \\
\hline \multicolumn{11}{|c|}{ Mixture C $\quad \mathbf{5 \%}$ methanol / $\mathbf{1 5 \%} \mathbf{O}_{2} / \operatorname{Ar}(\varphi=0.5)$} \\
\hline 1037 & 2 & \multicolumn{2}{|c|}{1500} & \multicolumn{2}{|c|}{562} & 130 & 573 & 1468 & 193 & Homogeneous \\
\hline 1186 & 2.3 & \multicolumn{2}{|c|}{1525} & \multicolumn{2}{|c|}{$\mathrm{F}^{*}$} & 0 & 621 & 1465 & 122 & Homogeneous \\
\hline \multicolumn{11}{|c|}{ Mixture D $\quad 5 \%$ ethanol $/ \mathbf{1 5 \%} \mathrm{O}_{2} / \operatorname{Ar}(\varphi=1)$} \\
\hline 1049 & 2.6 & \multicolumn{2}{|c|}{1190} & \multicolumn{2}{|c|}{$\mathrm{F}^{*}$} & 31 & 559 & 1613 & 69 & Homogeneous \\
\hline 1213 & 2.5 & \multicolumn{2}{|c|}{1326} & \multicolumn{2}{|c|}{$\mathrm{F}^{*}$} & 0 & 599 & 1601 & 59 & Homogeneous \\
\hline \multicolumn{11}{|c|}{ Mixture E $\quad 5 \%$ ethanol $/ 30 \% \mathrm{O}_{2} / \mathrm{Ar}(\varphi=0.5)$} \\
\hline 969 & 2.7 & 28 & 1772 & 26 & 1315 & $0,>208$ & 540 & 1624 & 2370 & Intermediate \\
\hline 1012 & 2.4 & 63 & 1730 & 39 & 1845 & 73 & 550 & 1618 & 1907 & Intermediate \\
\hline 1016 & 2.8 & 58 & $\mathrm{~F}^{*}$ & 35 & 1700 & 51,197 & 551 & 1622 & 1773 & Intermediate \\
\hline 1032 & 2.5 & 40 & 1473 & 45,72 & $\mathrm{~F}^{*}$ & 45,117 & 555 & 1618 & 656 & Intermediate \\
\hline 1076 & 2.5 & \multicolumn{2}{|c|}{1664} & \multicolumn{2}{|c|}{$\mathrm{F}^{*}$} & 20 & 567 & 1615 & 160 & Homogeneous \\
\hline 1081 & 2.3 & \multicolumn{2}{|c|}{2286} & \multicolumn{2}{|c|}{$\mathrm{F}^{*}$} & 16 & 568 & 1612 & 66 & Homogeneous \\
\hline
\end{tabular}

$\dot{x}$ : Measured front speed (axial)

$\mathbf{v}$ : Measured front speed (radial)

$\mathbf{x}_{\mathbf{0}}$ : Location of ignition from endwall

$\tau_{\mathrm{nh}}$ : Time between the appearance of first feature and main ignition

$\mathbf{F}^{*}$ : Fast ignition, speed unmeasurable due to spatial and temporal resolution

Uncertainty of measured speeds is $\pm 10 \%$ due to spatial and temporal resolution constraints

A key observation obtained by the lateral imaging is that lower temperatures incite the initial ignition to happen far from the endwall. This tendency is detected for all of the mixtures studied herein. Yamashita et al. [14] made similar observations and asserted that far-wall ignition may be caused by hot spots formed due to the interaction of reflected shock wave with boundary layer. Figure 5 shows the location of ignition $\left(\mathrm{x}_{0}\right)$ as a function of temperature for various mixtures investigated here. It is observed that the ignition 
takes place farther down the driven section at lower temperatures. For a given temperature, it is seen that methanol mixtures ignite farther from the endwall compared to ethanol mixtures, which indicates a higher susceptibility to far-wall ignition for methanol than ethanol.

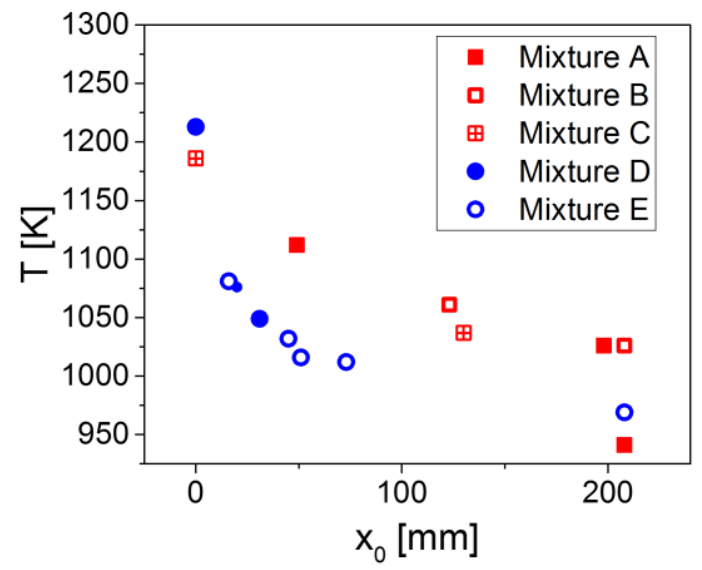

Figure 5. Dependence of ignition location on temperature for methanol (red) and ethanol (blue) mixtures.

At low temperatures, ignition delay time is relatively long and the residence time of hot spots will become relevant in the ignition modes. To depict this, we introduce a non-homogeneity characteristic time, $\boldsymbol{\tau}_{\mathbf{n h}}$, defined as the time between the appearance of the first emission feature as seen from the endwall and the time of the main ignition as detected by a strong rise in the pressure signal measured by the sidewall pressure transducer (20 $\mathrm{mm}$ from the endwall). The definition of $\boldsymbol{\tau}_{\mathbf{n h}}$ is graphically illustrated in Fig. 6 , and its values are listed in Table 2 for the various cases studied here. This parameter indirectly contains information about (i) the distance an ignition front would need to travel to arrive at the pressure transducer position, (ii) the speed of the ignition front, and (iii) the chemical nature of the mixture, related to the overall ignition delay time. As confirmed by the images, the lean $(\varphi=0.5)$ ethanol mixture E displays the highest $\boldsymbol{\tau}_{\mathbf{n h}}$ in comparison to other ethanol and methanol mixtures. At higher temperatures, $\boldsymbol{\tau}_{\mathbf{n h}}$ is significantly smaller since there is a greater chance of strong homogeneous ignition taking place near the 
endwall. This parameter is, therefore, an indication of the susceptibility of a fuel mixture to nonhomogenous ignition at different conditions.

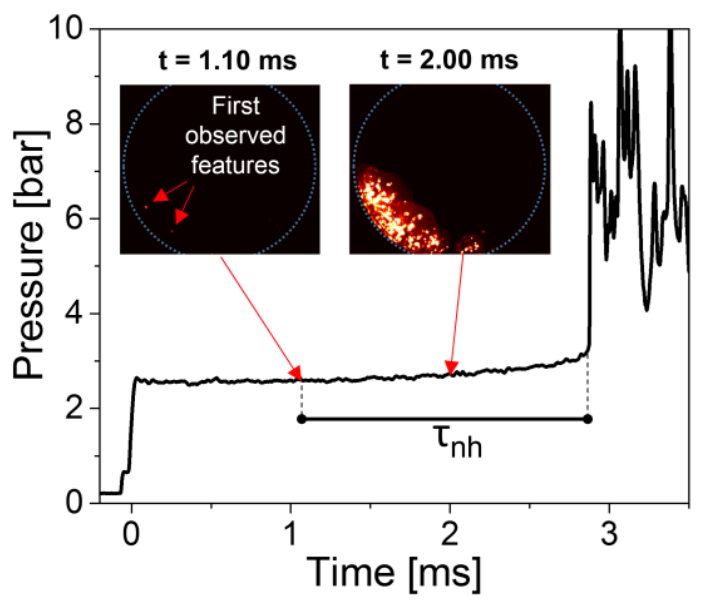

Figure 6. Definition of the non-homogeneity characteristic time $\tau_{\mathrm{nh}}$. Time lapse between the observation of first emission feature by endwall imaging and the main ignition as detected by the sidewall pressure trace. Example shown is for ethanol mixture $\mathrm{E}$ at $1016 \mathrm{~K}$ and $2.8 \mathrm{bar}$.

In general, by comparing many imaging results of ethanol and methanol, we may conclude that localized/non-homogeneous features appear significantly earlier for ethanol than for methanol. This observation correlates well with the low value of laminar flame thickness of mixture E. Although laminar flame thickness of mixture A is similar to this lean ethanol mixture, the methanol/air mixture exhibited less tendency for preignition. Therefore, laminar flame thickness may not be sufficient to explain preignition propensity of mixtures across various fuels. Alternatively, Sankaran et al. [28] proposed a criterion to identify ignition regime of a system as weak or strong, taking into consideration the laminar flame speed of the mixture, the temperature gradients in the system and the sensitivity of ignition delay time with temperature. This theory may be applied to further understand the tendency to preignite of the studied mixtures and will be explored in a future work.

The effect of the bath gas was analyzed by performing stoichiometric methanol experiments with argon and nitrogen. As seen in Table 2, mixtures with $\operatorname{Ar}$ (mixture A) and $\mathrm{N}_{2}$ (mixture B) behaved quite similar 
in terms of homogeneity and far-wall ignition propensity. By comparing the two experiments at $1026 \mathrm{~K}$ for the two methanol mixtures, respectively, we can observe that the propagation velocity of the initial features is higher in the Ar mixture compared to the $\mathrm{N}_{2}$ mixture. This may be correlated to the higher flame speed of the Ar mixture.

Both mixture $\mathrm{C}$ and $\mathrm{D}$ exhibited similar behavior in terms of the non-existence of initial slow features, and homogeneous ignition is observed with fast and strong emission fronts. At lower temperatures, farwall ignition was observed but the overall ignition remained largely homogeneous. As reported in Table 2, emission features of these two mixtures, in general, occurred closer to the endwall compared to other mixtures.

In accordance with the description of observed phenomena from the dual-camera imaging, the studied cases were classified into three categories:

1. Localized/non-homogeneous: An emission kernel is generated at a specific radial/axial position and it propagates with an initial subsonic speed until it provokes the ignition of the unburned mixture. Such cases are characterized by long $\boldsymbol{\tau}_{\mathbf{n h}}$ and a tendency for far-wall ignition. Example in Fig. 4.

2. Intermediate: A localized slow developing kernel is initially formed, but a secondary kernel with a faster supersonic propagation speed appears to ignite the mixture. Typified in both cases of Fig. 3.

3. Homogeneous: Characterized by a radially distributed supersonic fast feature, a short $\boldsymbol{\tau}_{\mathrm{nh}}$ and ignition location $\left(\mathrm{x}_{0}\right)$ close to zero (endwall). Example in Fig. 7. 


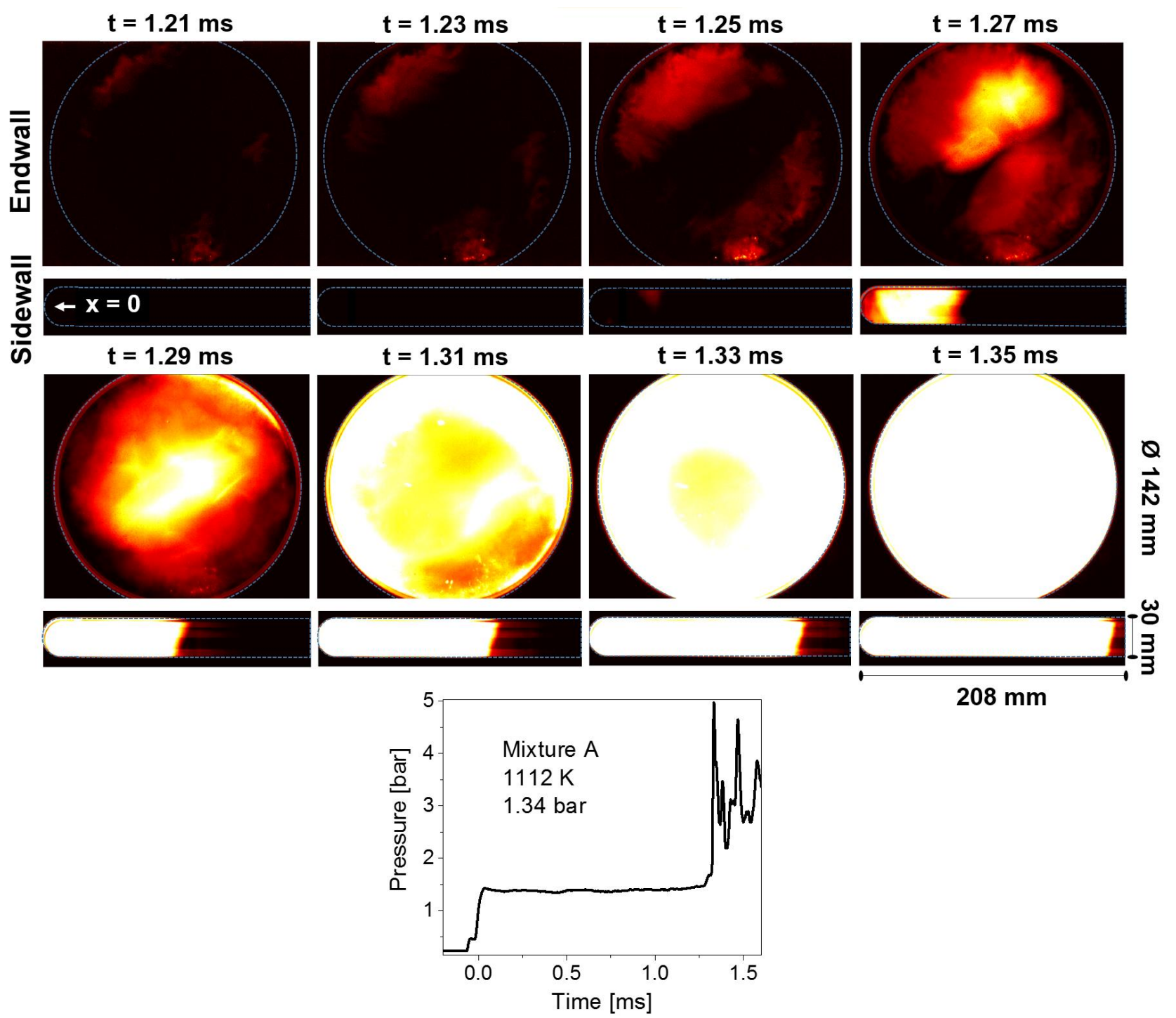

Figure 7. Simultaneous endwall and sidewall imaging of methanol mixture $\mathrm{A}$ at $1112 \mathrm{~K}$ and $1.3 \mathrm{bar}$. Indicated times refer to the time after the passage of reflected shock wave $(t=0)$. Endwall is located at $x=0$. Pressure trace measured with a Kistler pressure transducer at a sidewall port ( $2 \mathrm{~cm}$ from endwall) is also shown. This case is an example of an overall homogeneous ignition.

In order to elucidate the effect that the observed non-idealities have on ignition delay times (IDTs), Fig. 8 compares measured and simulated IDTs of the mixtures analyzed herein. A general tendency is observed, where the measured IDTs deviate from the predicted IDTs at lower temperatures. San Diego Mech [29] is used to compute IDTs of methanol mixtures, while AramcoMech [26] is used for ethanol mixtures. The methanol experiment at $941 \mathrm{~K}$ (mixture A), showing the largest deviation in Fig. 8a, was characterized in 
imaging experiments by the observation of localized ignition occurring far from the endwall and a long $\tau_{\mathrm{nh}}$. Ethanol mixtures, Fig. $8 \mathrm{~b}$, start to exhibit deviation from the model at higher temperatures compared to the methanol mixtures. Ethanol mixture $\mathrm{E}$ tests were characterized in imaging experiments by long $\tau_{\mathrm{nh}}$, observation of far-wall ignition and the formation of multiple emission fronts - factors which result in a large deviation from the modeled results. A thorough exploration of the IDTs of these mixtures, analysis of pressure traces and the observation of non-idealities will be the subject of a future work.

a)

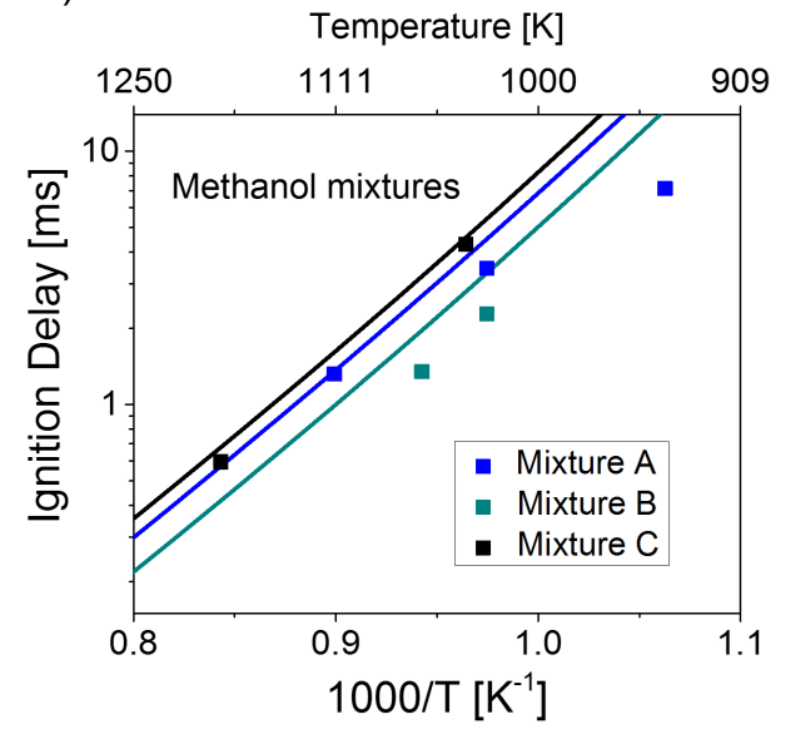

b)

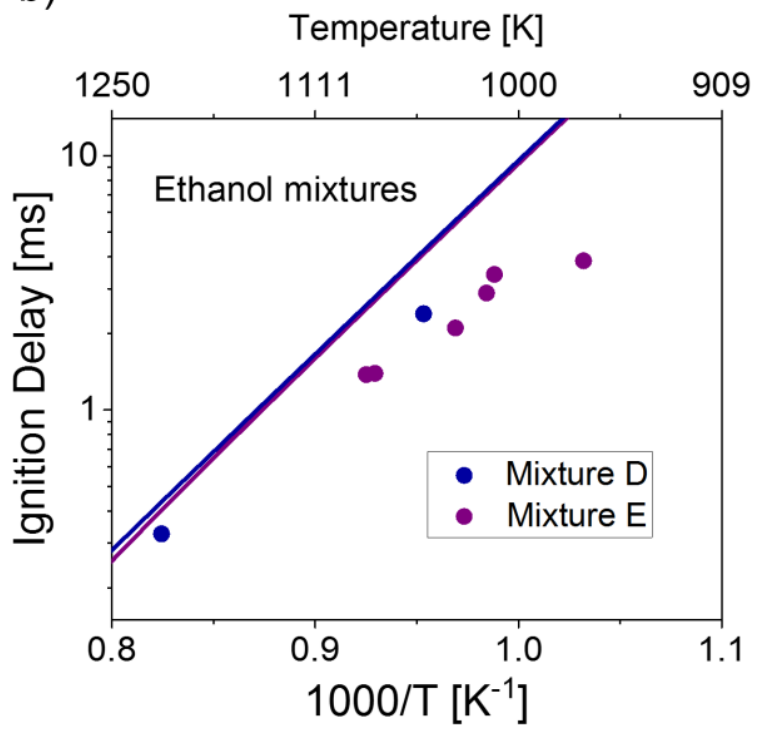

Figure 8. Ignition delay times of methanol and ethanol mixtures. Solid lines are constant volume simulations using a) San Diego Mech [29] at 1.5, 2 and 2.2 bar for mixture A, B and C, respectively, and b) AramcoMech

[26] model at 2.2 bar.

\subsection{Imaging of $\boldsymbol{n}$-Hexane Ignition}

Javed et al. [1] observed that ignition delay times of $n$-hexane were affected by localized ignition, where it was shown that the expedited ignition may have been promoted by the possible existence of hot spots. As $n$-hexane is well-studied in experiments and modelling, it may be argued that the relatively high fuel concentration (5\%) used by Javed et al. [1] affected the ignition of n-hexane. Therefore, in this work, dualcamera imaging experiments were performed for two stoichiometric mixtures of $n$-hexane, one with 
significantly higher concentration than the other, 5\% $n$-hexane/47.5\% $\mathrm{O}_{2} / \mathrm{Ar}$ and $1 \% n$-hexane/9.5\% $\mathrm{O}_{2} /$ Ar. Figure 9 compares the ignition of both mixtures at conditions where these have nominally similar ignition delay times. It may also be noted that these two conditions have similar Damköhler bifurcation number $\left(\mathrm{Da}_{\mathrm{bif}}=0.72\right.$ and 0.76 for $5 \%$ and $1 \% n$-hexane mixtures, respectively), a non-dimensional parameter proposed by Grogan and Ihme [5], which is a ratio of bifurcation and ignition timescales. Therefore, both conditions may be expected to have similar tendency of non-homogeneous ignition under this criterion, i.e., if bifurcation is the main source of inhomogeneity in these cases.

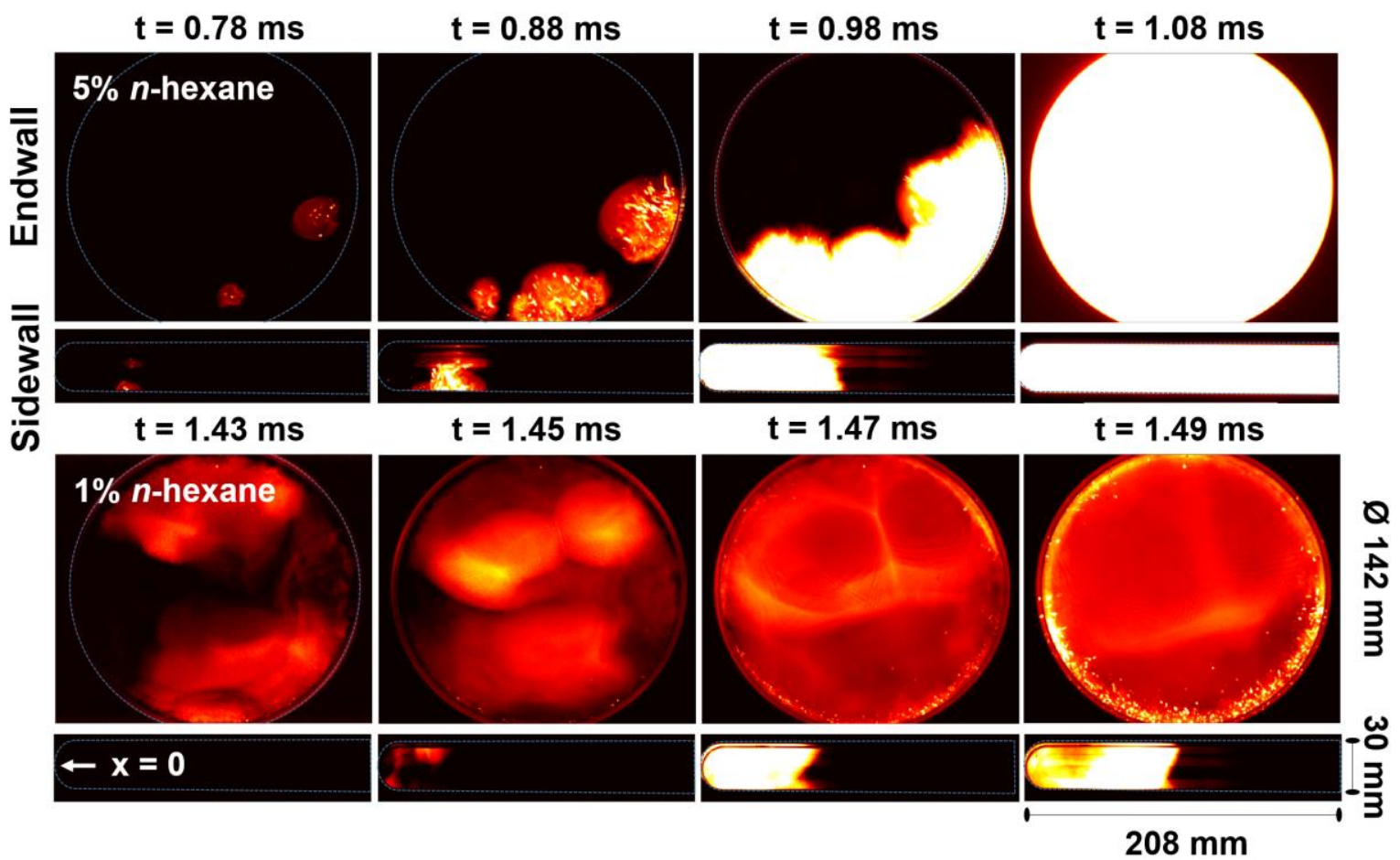

Figure 9. Simultaneous endwall and sidewall imaging of 5\% $n$-hexane/47.5\% $\mathrm{O}_{2} / \mathrm{Ar}(\varphi=1)$ at $994 \mathrm{~K}$ and 2.4 bar; $1 \% n$-hexane $/ 9.5 \% \mathrm{O}_{2} / \mathrm{Ar}(\varphi=1)$ at $1163 \mathrm{~K}$ and 2.4 bar. Indicated times for reach frame refer to the time after the passage of reflected shock wave $(\mathrm{t}=0)$. Endwall is located at $\mathrm{x}=0$.

The main difference between the ignition modes of these two mixtures is the homogeneity of the observed processes. The high-concentration n-hexane mixture ignited in a localized non-homogeneous manner 
whereas the low-concentration mixture, in contrast, exhibited a rapid and homogeneous process. The top panel of Fig. 9 shows an emission spot that can be observed at the right side of the circular section as early as $0.78 \mathrm{~ms}$, followed by a second and third feature from the bottom center and bottom left of the shock tube cross-section. These features grow and then join in a very energetic process that leads to the main ignition. Sidewall imaging reveals that the first feature appears at $48 \mathrm{~mm}$ from the endwall, and the main ignition front rapidly propagates from this location to both axial directions. The bottom panel of Fig. 9 shows the ignition of low-concentration mixture which depicts a relatively homogeneous process. From the endwall, one can identify two zones of emission at the top and the bottom that rapidly converge at the center of the circular section and thus lead to nearly homogeneous ignition of the mixture. The sidewall imaging shows that the ignition happens quite close $(\sim 4 \mathrm{~mm})$ to the endwall and develops in a nearly ideal manner. It may be noted that the emission intensity in this case is significantly smaller than that in the high-concentration case, which relates well to the energy content of each mixture. From these observations, we may conclude that $\mathrm{Da}_{\mathrm{bif}}$ is not sufficient to elucidate the differences of the observed ignition modes of the two studied $n$-hexane mixtures, favoring chemical sensitivity parameters rather than just a timescale competition, in contrast with the observations of Grogan and Ihme [5]

Table 3 summarizes the conditions tested for $n$-hexane mixtures. All experiments with $1 \% n$-hexane mixture exhibited fast homogeneous ignition and lateral imaging confirmed the appearance of ignition kernels very close to the endwall. This is translated in Table 3 as very short non-homogeneity characteristic times $\left(\boldsymbol{\tau}_{\mathbf{n h}}\right)$, ranging $43-246 \mu$ s, between the first emission feature and the observation of a supersonic front. These measured front speeds all fall significantly below the calculated $\mathrm{v}_{\mathrm{CJ}}$ for such conditions. The high-concentration mixtures, on the other hand, are characterized by an initial subsonic front which develops and grows until the main ignition is achieved. Lateral imaging for the case at $994 \mathrm{~K}$ and 2.4 bar (Fig. 9) shows that the ignition feature travels at a speed of $2085 \mathrm{~m} / \mathrm{s}$, which falls within $10 \%$ 
of the calculated $\mathrm{v}_{\mathrm{CJ}}$, thus providing the possibility of detonation waves in these conditions. The cases at $822 \mathrm{~K}$ and $882 \mathrm{~K}$ did not exhibit significant acceleration of the observed fronts and the propagation speeds remained low compared to both $\mathrm{v}_{\mathrm{CJ}}$ and $\mathrm{v}_{\text {sound, }}$, thus behaving as sequential autoignition.

Table 3. Summary of dual-camera imaging experiments: $n$-hexane

\begin{tabular}{|c|c|c|c|c|c|c|c|c|c|c|}
\hline \multirow[t]{2}{*}{$\mathrm{T}[\mathrm{K}]$} & \multirow{2}{*}{$\begin{array}{c}\mathrm{P} \\
{[\mathrm{bar}]}\end{array}$} & \multicolumn{2}{|c|}{$\dot{x}[\mathrm{~m} / \mathrm{s}]$} & \multicolumn{2}{|c|}{$\mathrm{v}[\mathrm{m} / \mathrm{s}]$} & \multirow[t]{2}{*}{$\mathrm{x}_{0}[\mathrm{~mm}]$} & \multirow{2}{*}{$\begin{array}{l}V_{\text {sound }} \\
{[\mathrm{m} / \mathrm{s}]}\end{array}$} & \multirow{2}{*}{$\begin{array}{c}\mathrm{v}_{\mathrm{CJ}} \\
{[\mathrm{m} / \mathrm{s}]}\end{array}$} & \multirow[t]{2}{*}{$\tau_{\mathrm{nh}}[\mu \mathrm{s}]$} & \multirow[t]{2}{*}{ Homogeneity } \\
\hline & & $\begin{array}{l}\text { First } \\
\text { feature }\end{array}$ & $\begin{array}{c}\text { At } \\
\text { ignition }\end{array}$ & $\begin{array}{c}\text { First } \\
\text { feature }\end{array}$ & $\begin{array}{c}\text { At } \\
\text { ignition }\end{array}$ & & & & & \\
\hline \multicolumn{11}{|c|}{$5 \%$ n-hexane / 47.5\% $\mathrm{O}_{2} / \mathrm{Ar}(\varphi=1)$} \\
\hline 822 & 2.7 & 155 & 300 & 66 & 95 & $141,>208$ & 471 & 1988 & 747 & Localized \\
\hline 882 & 2.7 & 234 & $\mathrm{~F}^{*}$ & 83 & 155 & 40,165 & 488 & 1984 & 780 & Localized \\
\hline 994 & 2.4 & 156 & 2085 & 30 & 895 & 48 & 516 & 1972 & 391 & Localized \\
\hline \multicolumn{11}{|c|}{$1 \%$ n-hexane $/ 9.5 \% \mathrm{O}_{2} / \operatorname{Ar}(\varphi=1)$} \\
\hline 1078 & 2.4 & \multicolumn{2}{|c|}{1149} & \multicolumn{2}{|c|}{1105} & $\sim 208$ & 579 & 1510 & 246 & Homogeneous \\
\hline 1163 & 2.4 & \multicolumn{2}{|c|}{1389} & \multicolumn{2}{|c|}{868} & 4 & 601 & 1505 & 43 & Homogeneous \\
\hline 1252 & 2.3 & \multicolumn{2}{|c|}{1259} & \multicolumn{2}{|c|}{$\mathrm{F}^{*}$} & 0 & 622 & 1499 & 79 & Homogeneous \\
\hline 1370 & 2.3 & \multicolumn{2}{|c|}{1199} & \multicolumn{2}{|c|}{$\mathrm{F}^{*}$} & 0 & 650 & 1492 & 59 & Homogeneous \\
\hline
\end{tabular}

$\dot{x}$ : Measured front speed (lateral)

v: Measured front speed (endwall)

$\mathbf{x}_{\mathbf{0}}$ : Location of ignition from endwall

$\tau_{\mathrm{nh}}$ : Time between the appearance of first feature and the main ignition

$F^{*}$ : Fast ignition, speed unmeasurable due to spatial and temporal resolution constraints

There are clearly different ignition mechanisms for the two $n$-hexane compositions, which clearly affect the measurements of ignition delays of these mixtures. Measured IDTs, deduced from sidewall measured pressure traces, of these mixtures are plotted in Fig. 10 and compared with the simulations of Zhang et al. [30] model. For the case of $1 \% n$-hexane, the measured IDTs agree well with the trend of the hightemperature region of the simulations, though the model slightly overestimates measured values. As noticed previously by Javed et al. [1], measured ignition delay times of the $5 \% n$-hexane/47.5\% $\mathrm{O}_{2} / \mathrm{Ar}$ mixture are significantly faster than predicted by the chemical kinetic model. 


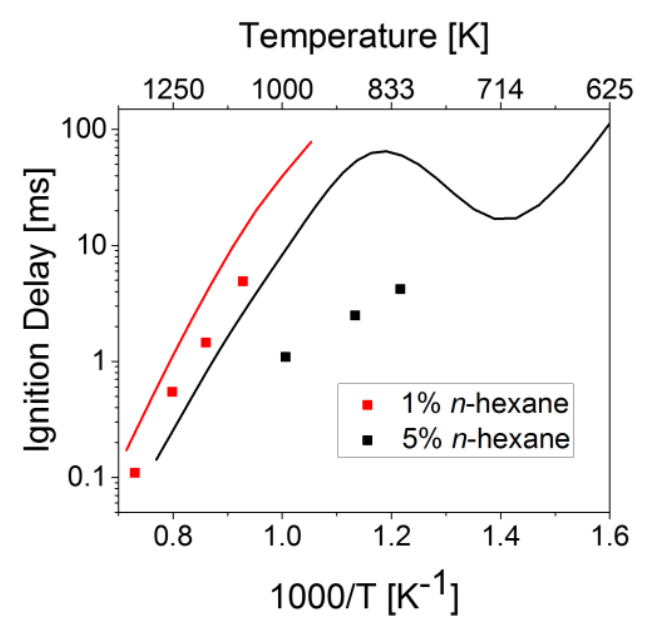

Figure 10. Ignition delay time measurements of 5\% $n$-hexane $/ 47.5 \% \mathrm{O}_{2} / \mathrm{Ar}$ and $1 \% n$-hexane/9.5\% $\mathrm{O}_{2} / \mathrm{Ar}$ for the cases studied with high-speed imaging (see Table 3). Solid lines depict constant volume simulations by Zhang et al. [30] model.

The two mixtures which clearly exhibited non-ideal ignition behavior in this work (lean 5\% ethanol and stoichiometric 5\% n-hexane) contained significantly higher amount of oxygen compared to other tested mixtures. High fuel concentration and/or high oxygen concentration increases the overall reactivity of the mixture, and seems to result in higher likelihood of hot spots and non-homogeneous ignition. Several works have looked into the non-ideal effects of shock wave interaction with boundary layer and shock bifurcation, which are more pronounced in driven gas mixtures containing high concentration of diatomic molecules. Grogan and Ihme [5] expect the existence of localized ignition close to the sidewall in bifurcation affected experiments. Yamashita et al. [14] describe the possibility higher temperature zones far from the endwall due to the acceleration of concave reflected shock waves caused by the interference with boundary layers. With tools like the dual-camera imaging diagnostic implemented in this work, identification of critical geometrical and combustion dynamics can be achieved and this can provide significant insights for experimental design and data evaluation.

\section{Conclusions}


Simultaneous sidewall and endwall high-speed imaging experiments were carried out in an optical end section of a circular shock tube. A comparison of ignition modes of methanol and ethanol showed that ethanol ignition kernels surge earlier in the test time, causing localized emission spots to be more influencing in the overall process, while the initial features observed in methanol are weaker and/or do not have sufficient time to affect the overall ignition homogeneity. Mixtures with both fuels have the tendency to ignite far from the endwall at lower temperatures. Experiments with $n$-hexane showed that a higher fuel concentration mixture tends to exhibit localized ignition kernels, which appear much earlier than the total ignition delay time and make the ignition process to be inhomogeneous, while a lower fuel concentration mixture exhibits a nearly ideal behavior with strong/fast ignition happening close to the endwall. Non-idealities characterized in this work are identified as factors affecting ignition delay time measurements.

Further work will focus on understanding the causes of hot spot formation and inhomogeneous ignition, and schlieren imaging will be implemented via the opposed slit windows to study the structure of shock waves, ignition fronts and detonations.

Supplementary Material: Imaging videos of the cases presented in the manuscript and a few others are included as Supplementary Material.

Acknowledgements: The paper is based on work supported by Saudi Aramco Research and Development Center FUELCOM program under Master Research Agreement Number 6600024505/01. FUELCOM (Fuel Combustion for Advanced Engines) is a collaborative research undertaking between Saudi Aramco and KAUST intended to address the fundamental aspects of hydrocarbon fuel combustion in engines, and develop fuel/engine design tools suitable for advanced combustion modes. 


\section{References}

[1] T. Javed, J. Badra, M. Jaasim, E. Es-Sebbar, M. Figueroa-Labastida, S.H. Chung, H.G. Im, A. Farooq, Shock Tube Ignition Delay Data Affected by Localized Ignition Phenomena, Comb. Sci. Tech. 189 (2017) 1138-1161.

[2] Y. Uygun, S. Ishihara, H. Olivier, A high pressure ignition delay time study of 2-methylfuran and tetrahydrofuran in shock tubes, Combust. Flame 161 (2014) 2519-2530.

[3] L.T. Pinzón, O. Mathieu, C.R. Mulvihill, I. Schoegl, E.L. Petersen, Ignition delay time and $\mathrm{H}_{2} \mathrm{O}$ measurements during methanol oxidation behind reflected shock waves, Combust. Flame 203 (2019) 143-156.

[4] O. Mathieu, L.T. Pinzón, T.M. Atherley, C.R. Mulvihill, I. Schoel, E.L. Petersen, Experimental study of ethanol oxidation behind reflected shock waves: Ignition delay time and $\mathrm{H}_{2} \mathrm{O}$ laser-absorption measurements, Combust. Flame 208 (2019) 313-326.

[5] K.P. Grogan, M. Ihme, Regimes describing shock boundary layer interaction and ignition in shock tubes, Proc. Combust. Inst. 36 (2017) 2927-2935.

[6] J. Santner, S.S. Goldsborough, Hot-spot induced mild ignition: Numerical simulation and scaling analysis, Combust. Flame 209 (2019) 41-62.

[7] M. Figueroa-Labastida, J. Badra, A.M. Elbaz, A. Farooq, Shock tube studies of ethanol preignition, Combust. Flame 198 (2018) 176-185.

[8] V.A. Troutman, C.L. Strand, M.F. Campbell, A.M. Tulgestke, V.A. Miller, D.F. Davidson, R.K. Hanson, High-speed $\mathrm{OH}^{*}$ chemiluminescence imaging of ignition through a shock tube end-wall, Appl. Phys. B 122 (2016) 56.

[9] A.M. Tulgestke, S.E. Johnson, D.F. Davidson, R.K. Hanson, High-speed imaging of inhomogeneous ignition in a shock tube, Shock Waves 28 (2018) 1089-1095. 
[10] E. Ninnemann, B. Koroglu, O. Pryor, S. Barak, L. Nash, Z. Loparo, J. Sosa, K. Ahmed, S. Vasu, New insights into the shock tube ignition of $\mathrm{H}_{2} / \mathrm{O}_{2}$ at low to moderate temperatures using high-speed endwall imaging, Combust. Flame 187 (2018) 11-21.

[11] O. Pryor, S. Barak, B. Koroglu, E. Ninnemann, S.S. Vasu, Measurements and interpretation of shock tube ignition delay times in highly $\mathrm{CO}_{2}$ diluted mixtures using multiple diagnostics, Combust. Flame 180 (2017) 63-76.

[12] K. Fieweger, R. Blumenthal, G. Adomeit, Self-ignition of S.I. engine model fuels: A shock tube investigation at high pressure, Combust. Flame 109 (1997) 599-619.

[13] C. Lee, S. Vranckx, A. Heufer, S. Khomik, Y. Uygun, H. Olivier, R. Fernandes, On the Chemical Kinetics of Ethanol Oxidation: Shock Tube, Rapid Compression Machine and Detailed Modeling Study, Z. Phys. Chem. 226 (2012) 1-28.

[14] H. Yamashita, J. Kasahara, Y. Sugiyama, A. Matsuo, Visualization study of ignition modes behind bifurcated-reflected shock waves, Combust. Flame 159 (2012) 2954-2966.

[15] A.C. Merkel, G. Ciccarelli, Visualization of lean methane-air ignition behind a reflected shock wave, Fuel 271 (2020) 117617.

[16] B.L. Wang, H. Olivier, H. Grönig, Ignition of shock-heated H2-air-steam mixtures, Combust. Flame 133 (2003) 93-106.

[17] E. Ninnemann, O. Pryor, S. Bara, S. Neupane, S. Vasu, High-speed 4-D Imaging Study of Isooctane Combustion in a Shock Tube, AIAA Scitech Forum (2019), paper 0119.

[18] D. Nativel, P. Niegemann, J. Herzler, M. Fikri, C. Schulz, A Study of Ethanol Oxidation in HighPressure Shock Tube: Ignition Delay Time Measurements and High-speed Imaging of the Ignition Process, 27th International Colloquium on the Dynamics of Explosions and Reactive Systems (2019), paper 386. 
[19] M. Figueroa-Labastida, A. Farooq, Simultaneous lateral and endwall high-speed visualization of ignition in a circular shock tube, Combust. Flame 214 (2020) 263-265.

[20] G.T. Kalghatgi, D. Bradley, Pre-ignition and 'super-knock' in turbo-charged spark-ignition engines, Int. J. Eng. Res., 13 (2012) 399-414

[21] A.R. Laich, E. Ninnemann, S. Neupane, R. Rahman, S. Barak, W.J. Pitz, S.S. Goldsborough, S.S. Vasu, High-pressure shock tube study of ethanol oxidation: Ignition delay time and CO time-history measurements, Combust. Flame 212 (2020) 486-499.

[22] R.D. Büttgen, T. Raffius, G. Grünefeld, H.-J. Koß, A. Heufer, High-speed imaging of the ignition of ethanol at engine relevant conditions in a rapid compression machine, Proc. Combust. Inst. 37 (2019) $1471-1478$.

[23] C. Wadkar, P. Chinnathambi, E. Toulson, An Experimental Study on the Factors Affecting Ethanol Ignition Delay Times in a Rapid Compression Machine, SAE Technical Paper (2019) 2019-01-0576.

[24] Y. Wang, Y. Qi, W. Liu, Z. Wang, Investigation of methanol ignition phenomena using a rapid compression machine, Combust. Flame 211 (2020) 147-157.

[25] S.M. Walton, He X., B.T. Zigler, M.S. Wooldridge, A. Atreya, An experimental investigation of isooctane ignition phenomena, Combust. Flame 150 (2007) 246-262

[26] C-W. Zhou, Y. Li, U. Burke, C. Banyon, K.P. Somers, S. Ding, S. Khan, J.W. Hargis, T. Sikes, O. Mathieu, E.L. Petersen, M. AlAbbad, A. Farooq, Y. Pan, Y. Zhang, Z. Huang, J. Lopez, Z. Loparo, S.S. Vasu, H.J. Curran, An experimental and chemical kinetic modeling study of 1,3-butadiene combustion: Ignition delay time and laminar flame speed measurements, Combust. Flame 197 (2018) 423-438.

[27] P. Niegemann, M. Fikri, S.A. Kaiser, C. Schulz, Ignition of Individual Droplets in a Reactive Fuel/Air Mixture behind Reflected Shock Waves, SAE International, 2019. 
[28] R. Sankaran, Im H.G., E.R. Hawkes, Chen J.H. The effects of non-uniform temperature distribution on the ignition of a lean homogeneous hydrogen-air mixture. Proc. Combust. Inst., 30 (2005) 875882.

[29] Chemical-kinetic mechanisms for combustion applications, Mechanical and Aerospace Engineering (Combustion Research), University of California at San Diego, San Diego Mechanism web page. URL: https://web.eng.ucsd.edu/mae/groups/combustion/mechanism.html

[30] K. Zhang, C. Banyon, C. Togbé, P. Dagaut, J. Bugler, H.J. Curran, An experimental and kinetic modeling study of n-hexane oxidation, Combust. Flame 162 (2015) 4194-4207. 